\title{
The Effect of Perforation Size and Site on Graft Success and Hearing in Cartilage Tympanoplasty with Mastoidectomy
}

\author{
Mehmet Fatih Karakuş ${ }^{1}$, Süleyman Emre Karakurt² ${ }^{2}$ Mustafa Çolak ${ }^{3}$, Hacı Hüseyin Dere ${ }^{4}$ \\ ${ }^{1}$ Department of Otorhinolaryngology, Faculty of Medicine, Ordu University, Ordu, Turkey. \\ ${ }^{2}$ University of Health Sciences Izmir Tepecik Education and Research Hospital, Department of Otorhinolaryngology, Izmir, \\ Turkey. \\ ${ }^{3}$ Ankara City Hospital, Department of Otorhinolaryngology, Ankara, Turkey. \\ ${ }^{4}$ Department of Otorhinolaryngology, Faculty of Medicine, Yıldırım Beyazit University, Ankara, Turkey.
}

Received: 28 September 2020, Accepted: 10 December 2020, Published online: 31 December 2020 (C) Ordu University Institute of Health Sciences, Turkey, 2020

\begin{abstract}
Objective: Chronic otitis media (COM) treatment aims to obtain a dry middle ear mucosa as much as possible with medical treatment and to closure the perforation in the tympanic membrane with the help of various graft materials after the eradication of the disease. In the presence of perforation, the surface area of the tympanic membrane is decreases, which causes a decrease in the sound pressure in the middle ear and adversely affect hearing. At present, there is no globally accepted standardization of factors affecting anatomical success of the graft and hearing outcomes. In this study, the effect of perforation size and site in the tympanic membrane on anatomic success and hearing was investigated in cases where autogenic composite tragal cartilage graft material was used.

Methods: The patients were classified in groups with respect to the perforation site (central or marginal) and size (large if the perforation comprised more than 50\% of the membrane area, and small if it comprised less) in the tympanic membrane. Anatomical success and preoperative-postoperative mean air bone gap pure tone average (ABG PTA) values of the graft were separately calculated for each group, and the ratios were compared.
\end{abstract}

Results: In 69 patients who underwent Type 1 tympanoplasty with mastoidectomy, 48 tympanic membrane perforations were central, 21 were marginal, 46 were small, and 23 were large. Graft anatomic success rates were $91.7 \%$ in the central group, $66.7 \%$ in the marginal group, $89.1 \%$ in the small group, and $73.9 \%$ in the large group. The anatomical success of the central group was found to be significantly higher than that of the marginal group. No difference was found between the small and large groups. When the effect on hearing was calculated, the postoperative hearing levels were significantly better in the central group.

Conclusion: Perforation size had no effect on the anatomical success and hearing level of the graft, while the perforation site affected both the anatomical success of the graft and the hearing level.

Key words: Tympanoplasty, cartilage, hearing, tympanic membrane perforation, mastoidectomy.

Suggested Citation: Karakus MF, Karakurt SE, Colak M, Dere HH. The Effect of Perforation Size and Site on Graft Success and Hearing in Cartilage Tympanoplasty with Mastoidectomy. Middle Black Sea Journal of Health Science, 2020; 6(3):364-368.

Address for correspondence/reprints:

Mehmet Fatih Karakuş

Telephone number: +90 (542) 5841459

ORCID-ID 0000-0002-6264-5416
E-mail: mfkarakus@yahoo.com

DOI: $10.19127 / \mathrm{mbsjohs} .801416$

Note: This study was presented as an oral presentation at the 41 st Turkish National Congress of Otorhinolaryngology Head and Neck Surgery (13-17 November 2019, Antalya). 


\section{Introduction}

Chronic otitis media (COM) is an important clinical condition characterized by inflamation of the middle ear and mastoid air cells, manifested by tympanic membrane perforation, recurrent otorrhea, and hearing loss. In the presence of perforation in the tympanic membrane, the membrane surface area decreases; this causes a decrease in the sound pressure passing to the middle ear, thus adversely affecting hearing (Jalali et al., 2017). If active infection is found in the middle ear and mastoid cells, it should first be contained with medical treatment, and then, after obtaining as dry middle ear mucosa as possible, the perforation should be closed with the help of various graft materials (Kamath et al., 2013).

Type 1 tympanoplasty is the closure of the perforation in the tympanic membrane using autogenous graft materials in cases with intact and mobile ossicular chains. The cause, size, and site of the perforation, the experience of the surgeon, and the applied surgical method can affect the success of autografts to varying degrees (Westerberg et al., 2011).

In this study, the effect of perforation size and site in the tympanic membrane on anatomic success and hearing was investigated in cases where autogenic composite tragal cartilage graft material was used.

\section{Methods}

A total of 69 patients (both male and female) over the age of 18 who underwent type 1 tympanoplasty with mastoidectomy using a composite graft prepared from autogenous tragal cartilage to repair the perforation in the tympanic membrane in the Department of Otorhinolaryngology (ENT) of a Tertiary Training and Research Hospital between January 2015 and December 2018 were retrospectively evaluated. Compliance of the study with the principles of the Helsinki Declaration was approved by the local ethics committee (Ethics Committee Approval dated July 25, 2019, numbered E-19-025) and informed consent was obtained from all patients before the surgery. Anamnesis, microscopic examination, and high-resolution computed tomography (HRCT) findings were reviewed. The patients were divided into groups based on whether the perforation in the tympanic membrane was in the central or posterior-dominated marginal location and whether the perforation was large, covering more than $50 \%$ of the membrane area, or small, covering less than $50 \%$ membrane area (Westerberg et al., 2011). Patients with other accompanying inner ear-middle ear pathologies, such as cholesteatoma, glomus tympanicum, traumatic- nontraumatic ossicular chain disorders, anatomical malformation, and congenital anomaly, patients with tympanosclerosis and adhesive otitis, patients who were operated for revision purposes, and patients who did not undergo mastoidectomy were excluded from the study.

Pure-tone speech audiometry measurements were performed at the preoperative and postoperative 12th month. Air conduction (AC) threshold was measured at frequencies of 250,500,1000, 2000, 4000, and $6000 \mathrm{~Hz}$, and bone conduction (BC) threshold was measured at frequencies of 500, 1000, 2000, and 4000 Hz. Pure-tone averages (PTAs) were then determined based on the threshold values at 500, 1000, 2000 and $4000 \mathrm{~Hz}$, and air bone gap (ABG) PTA values were calculated. For audiometric tests, AC 40 clinical audiometers (Interacuostic, Denmark) were used. Temporal bone HRCT was performed on all patients during the preoperative period. Following a scan with 0.5 -mm axial and 1-mm coronal cross-sectional range using an Alexion multislice 16 CT scanner (Toshiba, Otawara City, Tochigi, Japan), 0.3-mm axial reconstruction was performed.

All operations were performed under general anesthesia, using a surgical microscope (MollerWedel Optical; Hamburg, Germany), under the supervision of surgeons with otologic surgical experience of at least 10 years, and using a retroauricular approach. The composite cartilage graft, which was prepared by elevating the unilateral perichondrium from the tragal cartilage without thinning, was placed with the underlay technique, with the perichondrum extended to the inferior wall of the external auditory canal. At postoperative 1st, 3rd, 6th, and 12th months, whether the tragal cartilage graft material was intact or perforated was evaluated with otoscopic and microscopic examinations. Anatomical success at the 12th month and preoperative-postoperative ABG PTA values were calculated for each group and the rates were compared among themselves.

\section{Statistical analysis}

Shapiro Wilk test was used to evaluate the distribution of data. Categorical data were expressed as percentage, and continuous data were expressed as mean and standard deviation. Categorical data were evaluated using the Chi-square test or Fisher's exact test. Differences between two independent groups were analyzed using the unpaired t-test or the MannWhitney U test. P $<0.05$ was considered statistically significant. SPSS statistics software (SPSS for Windows version 21.0; SPSS Inc., Chicago, IL, USA) was used for all statistical calculations. 


\section{Results}

In the study, $37(53.6 \%)$ of the 69 patients were male and $32(46.4 \%)$ were female, and the mean age of the patients was $34.0 \pm 12.6$ years (range: 18-59).

Furthermore, $48(69.6 \%)$ of the perforations in the tympanic membrane were central, 21 (30.4\%) were marginal with posterior localization, $46(66.7 \%)$ were small, and $23(33.3 \%)$ were large. Gender and age distributions of all the groups were similar.

At the end of one year, the graft was intact and at its normal position (anatomical success) in 44 $(91.7 \%)$ of the 48 patients in the central perforation group and in $14(66.7 \%)$ of the 21 patients in the marginal perforation group. When preoperativepostoperative ABG PTA values and anatomic success rates were compared, we found that patients with central perforation had significantly better preoperative $(\mathrm{p}=0.019)$ and postoperative hearing $(\mathrm{p}$ $<0.001)$ as well as anatomical success $(\mathrm{p}=0.027)$ (Table 1).

Anatomical success was achieved in $41(89.1 \%)$ of the 46 patients in the small perforation group and in $17(73.9 \%)$ of the 23 patients in the large perforation group. Similarly, when preoperativepostoperative ABG PTA values $(\mathrm{p}=0.132, \mathrm{p}=0.115)$ and anatomical success rates $(\mathrm{p}=0.161)$ were compared, no significant difference was found (Table 2).

Table 1: Findings according to tympanic membrane perforation site

\begin{tabular}{|c|c|c|c|c|}
\hline & & $\begin{array}{l}\text { Central Perforation } \\
\qquad(\mathrm{N}=48)\end{array}$ & $\begin{array}{l}\text { Marginal Perforation } \\
\qquad(\mathrm{N}=21)\end{array}$ & $p$ value \\
\hline \multicolumn{2}{|c|}{ Age (years) } & $33.6 \pm 11.7$ & $34.9 \pm 14.7$ & 0.839 \\
\hline \multirow{2}{*}{ Gender } & Male & $25(52.1 \%)$ & $12(57.1 \%)$ & \multirow{2}{*}{0.698} \\
\hline & Female & $23(47.9 \%)$ & $9(42.9 \%)$ & \\
\hline \multirow{2}{*}{ Graft } & Successful & $44(91.7 \%)$ & $14(66.7 \%)$ & \multirow{2}{*}{0.027} \\
\hline & Unsuccessful & $4(8.3 \%)$ & $7(33.3 \%)$ & \\
\hline \multicolumn{2}{|c|}{ ABG PTA $^{1}(\mathrm{~dB})$} & $23.7 \pm 7.6$ & $29.6 \pm 10.7$ & 0.019 \\
\hline \multicolumn{2}{|c|}{$\mathrm{ABG} \mathrm{PTA}^{2}(\mathrm{~dB})$} & $10.1 \pm 4.2$ & $20.2 \pm 9.1$ & $<0.001$ \\
\hline
\end{tabular}

The values indicate means and standard deviations, N: Number of ears, dB: Decibel

PTA: Pure Tone Average $(500,1000,2000$, and $4000 \mathrm{~Hz})$, ABG: Air Bone Gap, ABG PTA ${ }^{1}$ : Preoperative, ABG PTA $^{2}$ : Postoperative.

Table 2: Findings according to tympanic membrane perforation size

\begin{tabular}{|c|c|c|c|c|}
\hline & & $\begin{array}{l}\text { Small Perforation } \\
\quad(\mathrm{N}=46)\end{array}$ & $\begin{array}{l}\text { Large Perforation } \\
\quad(\mathrm{N}=23)\end{array}$ & $\mathrm{p}$ value \\
\hline \multicolumn{2}{|c|}{ Age (years) } & $32.2 \pm 12.0$ & $37.6 \pm 13.2$ & 0.111 \\
\hline \multirow{2}{*}{ Gender } & Male & $26(56.5 \%)$ & $11(47.8 \%)$ & \multirow{2}{*}{0.732} \\
\hline & Female & $20(43.5 \%)$ & $12(52.2 \%)$ & \\
\hline \multirow{2}{*}{ Graft } & Successful & $41(89.1 \%)$ & $17(73.9 \%)$ & \multirow{2}{*}{0.161} \\
\hline & Unsuccessful & $5(10.9 \%)$ & $6(26.1 \%)$ & \\
\hline \multicolumn{2}{|c|}{$\operatorname{ABG~PTA~}^{1}(\mathrm{~dB})$} & $24.0 \pm 8.9$ & $27.5 \pm 9.2$ & 0.132 \\
\hline \multicolumn{2}{|c|}{$\mathrm{ABG} \mathrm{PTA}^{2}(\mathrm{~dB})$} & $12.0 \pm 6.7$ & $15.6 \pm 8.9$ & 0.115 \\
\hline
\end{tabular}

The values indicate means and standard deviations, N: Number of ears, dB: Decibel

\section{Discussion}

The objective of type 1 tympanoplasty is to repair the perforation in the tympanic membrane, prevent recurrent ear discharge, middle ear infections, and improve hearing loss. This operation can be performed with or without mastoidectomy, depending on the extent of the pathology in the middle ear and mastoid cells.

McGrew et al. evaluated the effectiveness of mastoidectomy on the repair of uncomplicated tympanic membrane perforations and found that persistent otological disease and the need for a secondary surgical intervention in the same ear were almost 50\% less common in the group that underwent tympanoplasty in combination with mastoidectomy. In addition, even if there is no evidence of active disease, they recommended that tympanoplasty and mastoidectomy should be performed together during the repair of simple perforations and reported that their hearing results were also comparable (McGrew et al., 2004). In contrast, other studies have reported that tympanoplasty can be performed without mastoidectomy, and the anatomical success in the use of cartilage graft material is not affected by this 
situation (Mishiro et al., 2001; Oz et al., 2018). We use both methods in our clinic; however, in the present study, patients who underwent tympanoplasty together with mastoidectomy were evaluated.

Temporal fascia, tragal-conchal cartilage, perichondrium, and adipose tissue can be used as autogenous graft material in perforation repair (Jalali et al., 2017). Cartilage and perichondrium are mesenchymal originated formations. The cartilage has various advantages, including being easy to manipulate and maintaining its vitality for a long time. Using a perichondrium-supported graft (composite graft) as the graft material makes an important contribution to epithelialization in the tympanic membrane in the postoperative period (Levinson 1987, Milewski 1993). Zhang et al. compared three different autogenous graft materials in a patient group comprising 75 small perforations and 42 large perforations and found that graft success was the highest in the group in which tragal cartilageperichondrium composite grafts were used. Furthermore, they stated that the long-term benefits of this graft material, especially in the large perforation group, were better in terms of both hearing and tympanic membrane morphology (Zhang et al., 2011). Gamra et al. (2008) evaluated the anatomical and audiological results of type 1 cartilage tympanoplasty and stated that they achieved functional results similar to the temporal fascia. Moreover, they recommended the use of cartilage grafts as the first choice in tympanoplasty operations. In our clinic, in the last 5 years, cartilage graft materials are being used with an increasing frequency, especially in cases undergoing simultaneous mastoidectomy.

Karela et al. stated that both perforation site and size had no significant effect on graft success (Karela et al., 2008). In a different study reporting the results of type 1 tympanoplasty, graft success was $87.2 \%$ in cases with central perforation, $88.9 \%$ in cases with marginal perforation, $75.0 \%$ in the subtotal perforated group, and $50.0 \%$ in the total perforated group; in addition, it was reported that perforation site and size had no effect on anatomical success (Yilmaz et al., 2009). In the study of Westerberg et al., these rates were $98.5 \%$ in the central perforation group, $94.1 \%$ in the marginal perforation group, $92.0 \%$ in the small perforation ( $<25 \%$ perforation) group, $96.2 \%$ in the medium perforation group ( $25 \%-50 \%$ perforation), and $97.9 \%$ in the large perforation group $(>50 \%$ perforation), and they concluded that neither the site nor the size of the perforation had a significant effect on the success of the operation (Westerberg et al., 2011). A different study investigating the correlation of anatomical and functional results with prognostic factors in tragal cartilage tympanoplasty reported that in cases where the perforation size was more than $50 \%$ of the tympanic membrane area, the graft anatomic success rate was significantly lower $(\mathrm{Oz}$ et al., 2018). In this study, perforation size had no effect on graft success, and marginal perforation localization significantly reduced the success rate.

Alsarhan et al. (2016) investigated the relationship between the degree of hearing loss and perforation size and site by forming groups of small, moderate, and large perforations and dividing perforation sites into anteroinferior, anterosuperior, posteroinferior, and posterosuperior quadrants. They stated that hearing worsened as the perforation size increased and that hearing was worse in perforations located in the posteroinferior quadrant compared to those located in the other quadrants. When the preoperative ABG PTA values of our cases were reviewed, we found that there was no relationship between perforation size and hearing, and hearing was worse in the marginal perforation group with predominantly posterior quadrant localization.

In another study where the effects of a successful operation, the size and site of the perforation, and other patient criteria on mean AC (AC PTA) and ABG PTA parameters were investigated, both perforation size and site had an effect on postoperative hearing gain, with the highest gain in subtotal perforated and big central perforated groups (Dawood, 2017). Wasson et al. reported that the postoperative mean airway hearing gain was positively correlated with preoperative perforation size (Wasson et al., 2009). A different study stated that hearing was worse in patients with large perforation before the operation, and the level of recovery in these cases following the operation (hearing gain) was better than the hearing gain of patients with small perforation (Kumar, 2015). Contrary to these studies, Rasha and Ahmed (2015) reported that the postoperative hearing gain of patients with small perforations was better than that of patients with a large preoperative perforation size and poor hearing. In the present study, preoperative and postoperative hearing levels of patients with central perforation were better than those of patients with marginal perforation. Contrary to our expectations, hearing levels in both the preoperative and postoperative periods did not differ significantly difference in the large perforation group, where the perforation covered more than $50 \%$ of the tympanic membrane. 


\section{Conclusion}

In conclusion, we determined that perforation size had no effect on graft anatomic success and hearing level, while perforation site affected both. Following the calculation of surface area measurements of perforations in the tympanic membrane by endovision imaging systems, the results of the present study should be supported with larger case studies.

\section{Acknowledgment}

The authors thank Enago https://www.enago.com.tr/ceviri/ for their assistance in manuscript translation and editing.

Ethics Committee Approval: Ethics committee approval was received for this study from the ethics committee of Ministry of Health, Ankara City Hospital (Number: E-19-025, July 25, 2019).

Peer-review: Externally peer-reviewed.

Author Contributions: Conception: M.F.K Design: M.F.K, S.E.K Supervision: H.H.D Materials: M.F.K, S.E.K, M.C, H.H.D Data collection and/or processing: M.F.K, S.E.K Analysis and/or interpretation: M.F.K, S.E.K, M.C, H.H.D Literature review: M.F.K, M.C Writer: M.F.K Critical review: H.H.D.

Conflict of Interest: No conflict of interest was declared by the author.

Financial Disclosure: The author declared that this study hasn't received no financial support.

\section{References}

Alsarhan HE, Dawood MR, Jwery AAK, Khammas $\mathrm{AH}$, Hamad AK. Assessment of hearing loss in tympanic membrane perforation. Adv Arab Acad Audio-Vestibul Journal 2016; 3: 16-9.

Dawood MR. Hearing evaluation after successful myringoplasty. Journal of Otology 2017; 12 (4): 192-7.

Gamra OB, Mbarek C, Khammassi K, Methlouthi N, Ouni H, Hariga I, et al. Cartilage graft in type I tympanoplasty: audiological and otological outcome. Eur Arch Otorhinolaryngol 2008; 265(7): 739-42.

Jalali MM, Motasaddi M, Kouhi A, Dabiri S, Soleimani R. Comparison of cartilage with temporalis fascia tympanoplasty: A meta-analysis of comparative studies. Laryngoscope 2017; 127(9): 2139-48.

Kamath MP, Sreedharan S, Rao AR, Raj V, Raju K. Success of myringoplasty: our experience. Indian J Otolaryngol Head Neck Surg 2013; 65(4): 35862.
Karela M, Sandeep B, Watkins A, Phillipps JJ. Myringoplasty: surgical outcomes and hearing improvement: is it worth performing to improve hearing? Eur Arch Otorhinolaryngol 2008; 265(9): 1039-42.

Kumar TVVV. Evaluation of various graft materials in myringoplasty. Int J Pharm Bio Sci 2015; 6(1): 700-23.

Levinson RM. Cartilage-perichondrial composite graft tympanoplasty in the treatment of posterior marginal and attic retraction pockets. Laryngoscope 1987; 97(9): 1069-74.

McGrew BM, Jackson CG, Glasscock ME. Impact of mastoidectomy on simple tympanic membrane perforation repair. Laryngoscope 2004; 114(3): 506-11.

Milewski C. Composite graft tympanoplasty in the treatment of ears with advanced middle ear pathology. Laryngoscope 1993; 103(12): 1352-6.

Mishiro Y, Sakagami M, Takahashi Y, Kitahara T, Kajikawa H, Kubo T. Tympanoplasty with and without mastoidectomy for noncholesteatomatous chronic otitis media. Eur Arch Otorhinolaryngol 2001; 258(1): 13-5.

Öz I, Erbek SH, Erbek S, Özlüoğlu LN. Anatomic and functional results in tragal cartilage tympanoplasty in adults: Correlation with prognostic factors. Turkish Journal Of Ear Nose And Throat 2018; 28(2): 78-84.

Rasha A, Ahmed SAO. Outcome of hearing improvement in myringoplasty a study of fifty one Sudanese patients. International Journal of Otorhinolaryngology 2015; 1(1): 5-8.

Wasson JD, Papadimitriou CE, Pau H. Myringoplasty: impact of perforation size on closure and audiological improvement. J Laryngol Otol 2009; 123(9): 973-7.

Westerberg J, Harder H, Magnuson B, Westerberg L, Hydén D. Ten-year myringoplasty series: does the cause of perforation affect the success rate? J Laryngol Otol 2011; 125(2): 126-32.

Y1lmaz S, Karaman E, Güçlü E, Yaman H, Akkan N. Type 1 tympanoplasty results. Duzce Medical Journal 2009; 11(3): 33-6.

Zhang ZG, Huang QH, Zheng YQ, Sun W, Chen YB, $\mathrm{Si}$ Y. Three autologous substitutes for myringoplasty: a comparative study. Otol Neurotol 2011; 32(8): 1234-8. 